\title{
ON EQUICONTINUOUS TRANSFORMATION GROUPS
}

\author{
SABER ELAYDI
}

It is shown that, under certain conditions, a transformation group is pointwise equicontinuous if and only if it is $P$-equicontinuous for some replete semigroup $P$ in the phase group.

Let $(X, T, \pi)$ be a transformation group, where $X$ is assumed to be a locally compact $T_{2}$ uniform space with a prescribed uniformity $U$ and $T$ is a topological group. In [1] Baum gave a condition, using replete semigroups [3], for a transformation group $(X, T, \pi)$ with a compact phase space $X$ to be uniformly equicontinuous. The main purpose of this paper is to give a condition for pointwise equicontinuity for a wider range of transformation groups; namely those transformation groups with locally compact phase spaces. We use the notion of $P$-equicontinuity, where $P$ is a replete semigroup in $T$, which generalizes the notion of positive equicontinuity of Coven [2]. Our techniques depend heavily on the notions of prolongation which were lifted from the theory of dynamical systems to topological dynamics by Hajek [4]. These techniques are interesting in themselves and they might prove effective in dealing with several problems in topological dynamics.

For the convenience of the reader we give the definitions of the basic notions used in the paper. For $x \in X$, and a replete semigroup $P$ in $T$, we have the following definitions:

Received 16 June 1981. 
the $P$-limit set of $x$ :

$$
L^{P}(x)=\cap\{\overline{x p P} \mid p \in P\} ;
$$

the P-prolongation set of $x$ :

$$
{ }_{D}^{P}(x)=\cap\{\overline{V P} \mid V \text { is a neighborhood of } x\} \text {. }
$$

Note that one can extend the transformation group $(X, T, \pi)$ to $\left(X^{*}, T, \pi^{*}\right)$, where $X^{*}=X \cup\{\infty\}$ is the one-point compactification of $X$, by letting $\pi^{*}(x, t)=\pi(x, t)$ for each $x \in X$ and $t \in T$ and $\pi^{*}(\infty, t)=\infty$ for each $t \in T$. The corresponding notions in $\left(X^{*}, T, \pi^{*}\right)$, of $L^{P}(x)$ and $D^{P}(x)$ are denoted, respectively, by $L_{*}^{P}(x)$ and $D_{*}^{P}(x)$. The closure of a set $A$ in $X^{*}$ is denoted by $\bar{A}^{*}$. The boundary of $A$ is denoted by $\partial A$.

For breviety $\pi(x, t)$ is denoted by $x t$ and $(X, T, \pi)$ is written as $(X, T)$. Our main references for notations and terminology are [3] and $[4]$.

DEFINITION. Let $x \in X$ and let $P$ be a replete semigroup in $T$. Then $P$ is said to be equicontinuous at $x$ if for every $\alpha \in U$ there exists $\beta \in U$ such that wenever $(x, y) \in \beta$ then $(x p, y p) \in \alpha$ for each $p \in P$. A transformation group $(X, T)$ is said to be pointwise P-equicontinuous if $P$ is equicontinuous at each $x \in X$.

THEOREM. A transformation group $(X, T)$ is pointwise equicontinuous if and only if it is pointwise p-equicontinuous provided that either one of the following conditions is satisfied:

(1) $X$ is locally connected and $\overline{x T}$ is compact for each $x \in X$, or

(2) $\overline{x T}$ is compact and $\overline{(x, y) T}$ is connected for each $x, y \in X$.

Proof. Sufficiency. Assume that $(X, T)$ is pointwise P-equicontinuous. The proof consists of three parts.

- Part (1). We will show that for $x, y \in X, D(x, y)=\overline{(x, y) T}$ in the squared transformation group $(X x X, T)$. Let $(a, b) \in D^{P}(x, y)$. Then there are nets $\left\{\left(x_{i}, y_{i}\right)\right\}$ in $X x X$ and $\left\{p_{i}\right\}$ in $P$ such that 
$\left(x_{i}, y_{i}\right) \rightarrow(x, y)$ and $\left(x_{i}, y_{i}\right) p_{i} \rightarrow(a, b)$. Let $\alpha \in U$ and $\beta \in U$ with $\beta^{2} \subset \alpha$. Then $\left(x p_{i}, a\right)=\left(x p_{i}, x_{i} p_{i}\right)\left(x_{i} p_{i}, a\right) \in \beta^{2} \subset \alpha$ for all $i \geq i_{0}$. Hence $x p_{i} \rightarrow a$. Similarly, one can show that $y p_{i} \rightarrow b$. Consequently, $(a, b) \in \overline{(x, y) P}$. Hence $D^{P}(x, y) \subset \overline{(x, y) P}$. Since it is always true that $\overline{(x, y) P} \subset D^{P}(x, y)$, it follows that $D^{P}(x, y)=\overline{(x, y) P}$.

We now show that $\overline{(x, y) T}$ is minimal for each $(x, y) \in X x X$. Since $\overline{(x, y) T}$ is compact, $L^{P^{-1}}(x, y) \neq \varnothing$. Let $(c, d) \in L^{P^{-1}}(x, y)$. Then

$$
(c, d) \in \overline{(c, d) T} \subset L^{P^{-1}}(x, y) \subset D^{P^{-1}}(x, y) \text {. }
$$

Thus $(x, y) \in D^{P}(c, d)=\overline{(c, d) P}$. This implies that

$$
\overline{(x, y) T} \subset \overline{(c, d) T} \subset L^{P^{-1}}(x, y) \text {. }
$$

Let $(e, f) \in \overline{(x, y) T}$. Then $(e, f) \in L^{P^{-1}}(x, y)$. Therefore, as in the above argument, $\overline{(x, y) T} \subset \overline{(e, f) T}$ and consequently $\overline{(x, y) T}$ is minimal. Furthermore, since $L^{P}(x, y) \neq \emptyset$, it follows that

$$
\overline{(x, y) T}=\overline{(x, y) P}=L^{P}(x, y) \text {. }
$$

Let $(g, h) \in D(x, y)$. Then there are nets $\left(g_{i}, h_{i}\right) \in X x X$ and $\left\{t_{i}\right\}$ in $T$ such that $\left(g_{i}, h_{i}\right) \rightarrow(x, y)$ and $\left(g_{i}, h_{i}\right) t_{i} \rightarrow(g, h)$. For each $i$,

$$
\left(g_{i}, h_{i}\right) t_{i} \in \overline{\left(g_{i}, h_{i}\right) T}=\overline{\left(g_{i}, h_{i}\right) P}=D^{P}\left(g_{i}, h_{i}\right)
$$

It follows that

$$
(g, h) \in D^{P}(x, y)=\overline{(x, y) P}=\overline{(x, y) T} .
$$

Thus $D(x, y) \subset \overline{(x, y) T}$. Since $\overline{(x, y) T} \subset D(x, y), D(x, y)=\overline{(x, y) T}$.

Part (2). Assuming conditions (1) or (2) in the hypothesis of the theorem we show that $D_{*}(x, y)=\overline{(x, y) T^{*}}$ for each $(x, y) \quad X x X$.

(a) Assume that $\overline{x T}$ is compact and $X$ is locally connected. Suppose that for some $(x, y) \in X x X, D_{*}(x, y) \neq \overline{(x, y) T^{*}}$. Since 
$\overline{(x, y) T^{*}}=\overline{(x, y) T}$, it follows that $\infty \in D_{*}(x, y)$. Then there exists a compact neighborhood $U \subset X$ of $\overline{(x, y) T}$ and a neighborhood filter $\left\{V_{\alpha}\right\}$ of connected open neighborhoods of $\overline{(x, y) T}$ contained in $U$ and directed by inclusion such that $V_{\alpha} t_{\alpha} \notin U$ for some $t_{\alpha} \in T$. Since $V_{\alpha} t_{\alpha}$ is connected, there exists $\left(x_{\alpha}, y_{\alpha}\right) \in V_{\alpha}$ such that $\left(x_{\alpha}, y_{\alpha}\right) t_{\alpha} \in \partial U$ for each $\alpha$. Since $\partial U$, is compact, we may assume that $\left(x_{\alpha}, y_{\alpha}\right) t_{\alpha} \rightarrow(c, d) \in \partial U$. Suppose that $\left(x_{\alpha}, y_{\alpha}\right) \rightarrow(e, f) \in \overline{(x, y) T}$. Then

$$
(c, d) \in D(e, f)=\overline{(e, f) T} \subset \overline{(x, y) T}
$$

which is a contradiction. It follows that

$$
D_{*}(x, y)=D(x, y)=\overline{(x, y) T}=\overline{(x, y) T^{*}}
$$

for each $(x, y) \in X x X$.

(b) Assume now that $\overline{x T}$ is compact and $\overline{(x, y) T}$ is connected for each $x, y \in X$. We first show that $D_{*}(x, y)$ is connected. Suppose that $D_{*}(x, y)$ is not connected and $D_{*}(x, y)=A \cup B$ is a separation of $D_{*}(x, y)$. If $(x, y) \in A$, then $\overline{(x, y)} T^{*}=\overline{(x, y) T}$ being connected lies entirely in $A$. There exists an open neighborhood $U$ of $A$ such that $\bar{U}$ is a compact subset of $X x X$ and $\bar{U} \cap B=\varnothing$. Let $(a, b) \in B$. Then there exist nets $\left\{\left(x_{\alpha}, y_{\alpha}\right)\right\}$ in $U$ and $\left\{t_{\alpha}\right\}$ in $T$ such that $\left(x_{\alpha}, y_{\alpha}\right) \rightarrow(x, y)$ and $\left(x_{\alpha}, y_{\alpha}\right) t \rightarrow(a, b)$. Since $\overline{\left(x_{\alpha}, y_{\alpha}\right)^{T}}$ is connected, $\left(x_{\alpha}, y_{\alpha}\right) T \cap \partial U \neq \emptyset$ for each $\alpha$. Let $\left(a_{\alpha}, b_{\alpha}\right) \in\left(x_{\alpha}, y_{\alpha}\right)^{T} \cap \partial U$ and assume that $\left(a_{\alpha}, b_{\alpha}\right) \rightarrow(c, d) \in \partial U$. Then we can find a net $\left\{s_{\alpha}\right\}$ in $T$ such that $\left(x_{\alpha}, y_{\alpha}\right) s_{\alpha} \rightarrow(c, d)$. Hence $(c, d) \in D(x, y) \cap \partial U$ and we thus have a contradiction. Therefore $D_{*}(x, y)$ is connected. If

$$
D_{*}(x, y) \neq \overline{(x, y)} T^{*}=\overline{(x, y) T},
$$

then

$$
D_{*}(x, y)=D(x, y) U\{\infty\}=\overline{(x, y) T} U\{\infty\} \text {. }
$$

This gives a separation of $D_{*}(x, y)$ into two nonempty disjoint closed sets and we have a contradiction. Consequently, $D_{*}(x, y)=\overline{(x, y) T}$ for each $(x, y) \in X x X$. 
Part (3). We now show that $T$ is equicontinuous at each point $x \in X$. Assume that $T$ is not equicontinuous at a point $x \in X$. Then there are nets $\left\{x_{i}\right\},\left\{y_{i}\right\}$ in $X$ and $\left\{t_{i}\right\}$ in $T$ and $\alpha \in U$ such that $\lim x_{i}=x=\lim y_{i}$ and $\left(x_{i} t_{i}, y_{i} t_{i}\right) \vDash \alpha$ for all $i$. Assume that $\left(x_{i}, y_{i}\right) t_{i} \rightarrow(a, b) \in X^{*} x X^{*}$. Then $(a, b) \in D_{*}(x, x)$. It follows from Part (2) that $(a, b) \in \overline{(x, x) T^{*}}=\overline{(x, x) T}$. This implies that $a=b \in X$ and we thus have a contradiction. This completes the proof of the sufficiency. The necessity is clear. The proof of the theorem is now complete.

We now give an example to show the sharpness of our theorem.

EXAMPLE. Let $(x, t)$ be a transformation group, where $X=(0,1]$ the half open unit interval and $T=\mathbb{R}$, the additive group of real numbers, acting on $X$ by the law $x t=2^{t} x /\left(\left(2^{t}-1\right) x+1\right)$. Then for each $x \in(0,1), x t>x$ if $t>0$ and $x t<x$ if $t<0$ and if $t, t^{\prime}$ are positive with $t<t^{\prime}$, then $x t<x t^{\prime}$. The point 1 is a fixed point. The transformation group $(X, T)$ is pointwise $\mathbf{R}^{+}$-equicontinuous but not pointwise equicontinuous, since $\mathbb{R}$ fails to be equicontinuous at the point 1 . Notice that none of the points in $(0,1)$ has a compact closure.

\section{References}

[1] John D. Baum, "An equicontinuity condition in topological dynamics", Proc. Amer. Math. Soc. 12 (1961), 30-32.

[2] Ethan M. Coven, "P-recursion and transformation groups having an equicontinuous replete semigroup", Math. Systems Theory 3 (1969), $170-178$.

[3] Walter Helbig Gottschalk and Gustav Arnold Hedlund, Topological dynamics (American Mathematical Society Colloquium Publication, 36. Amermican Mathematical Society, Providence, Rhode Island, 1955). 
[4] Otomar Hajek, "Prolongations in topological dynamics", Seminar on differential equations and dynomical systems, II, 79-89 (Seminar Lectures, University of Maryland, 1969. Lecture Notes in Mathematics, 144. Springer-Verlag, Berlin, Heidelberg, New York, 1970).

Department of Mathematics,

Kuwait University,

PO Box 5969,

Kuwait. 